\title{
Climatic modes of variability over the Holocene: Model-data synergies to improve future projections
}

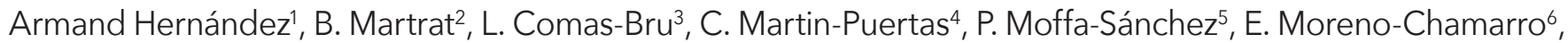
P. Ortega ${ }^{6}$ and D. Swingedouw ${ }^{7}$

Barcelona, Spain, 25-27 September 2019

The spatial structure of climate variability follows recurrent patterns, often referred to as modes of climate variability. The modes are suitable tools for understanding regional climate patterns on different spatio-temporal scales, which are in turn closely related to energy production, food security, and key ecosystem services like oceanic and terrestrial $\mathrm{CO}_{2}$ uptake and water availability (e.g. Jerez et al. 2013; Bastos et al. 2016; Zubiate et al. 2017). It is thus essential to improve our understanding of the evolution of climate modes of variability and their interactions at a global scale during the current interglacial period (i.e. the Holocene, covering the past 11,700 years). This is crucial to: (i) understand the potential variability of these modes and estimate their response to external forcing at the wide range of temporal scales, and (ii) evaluate the ability of different climate models to reproduce them robustly, given that identifying the most reliable simulations narrows down uncertainty in future nearterm fluctuations in climate and associated hazards.

For three days, 25 participants - including seven early-career researchers - from 11 countries of four continents shared their expertise in the research field of modes of variability during the first joint workshop of CLIMOVAR (CLImatic MOdes of VARiability) and IBCC-lo2k (IBerian Climate Change paleoarchive - synthesis and stewardship of land-ocean data, taking the past 2000 years as a reference). CLIMOVAR, emphasizing the atmospheric/oceanic dimension of the climate modes, aligns well with the IBCC-lo2k initiative, which synthesizes and preserves the climate measurements of the past, integrating land and ocean data of the Iberian

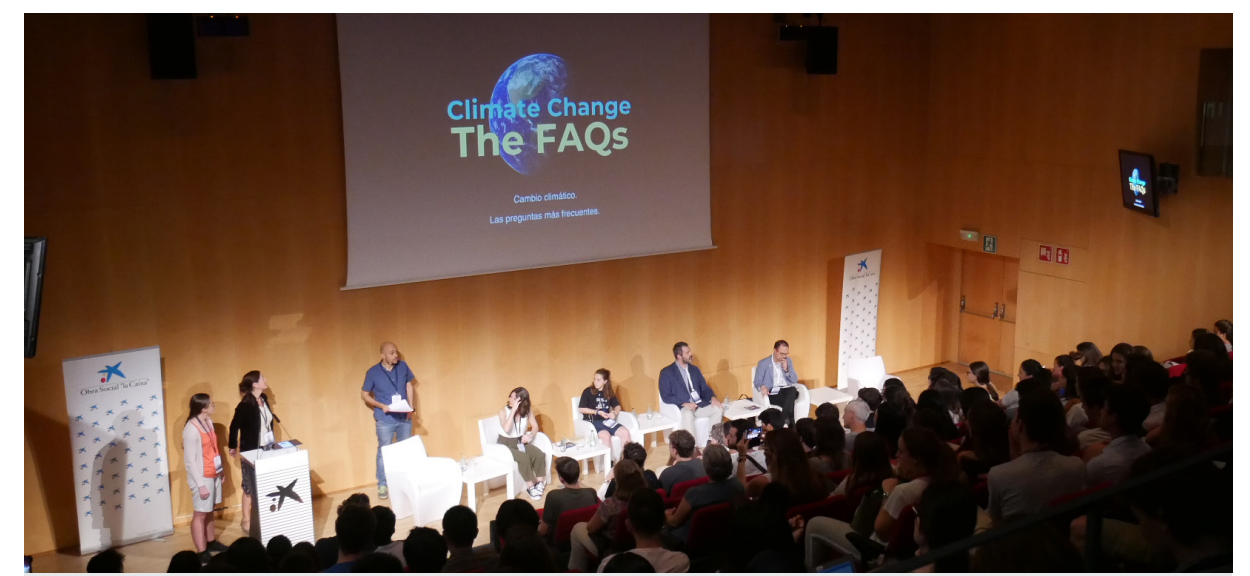

Figure 1: Armand Hernández, leader of the workshop organizing team, presents the video "Climate Change: the FAQs" to the outreach event audience (comprised primarily of secondary school students) at the end of the

CLIMOVAR meeting. Photo credit: Jordi Cortés.

region. In this sense, the meeting provided a unique opportunity to explore synergies between paleoclimatologists using natural proxy archives, paleoclimate modelers, and statisticians, in order to better evaluate prevailing atmospheric and oceanic circulation modes on interannual-to-centennial timescales.

The CLIMOVAR/IBCC-lo2k team discussed five main topics of broad interest to the community: (i) describing climate modes of variability during the Holocene; (ii) bridging the gaps between models and climate proxy records; (iii) deciphering the role of external forcing on climate modes of variability; (iv) evaluating the impacts of climate modes of variability; and ( $v$ ) assessing potential synergies with existing PAGES working groups. All topical sessions included short talks for participants to present their work and subsequent exciting discussions. Moreover, two keynote talks, discussion panels, and an outreach event (Fig. 1) completed the large variety of individual contributions.

The workshop allowed us to highlight that modes of variability are different from mean-state changes, so that it is necessary to first define a mean state for a given period before correctly defining variability modes. The general consensus was to apply for a new PAGES working group with the following specific goals: (i) focus on the Holocene time windows also covered by modeling initiatives like the Paleoclimate Modelling Intercomparison Project (PMIP4); (ii) use pseudo-proxies from models to test whether it is possible to reliably reconstruct modes of variability during the selected Holocene time windows with the available proxy data and produce the actual reconstructions that are validated by the models; (iii) screen existing databases to find high-quality records necessary to reconstruct variability modes using state-of-the-art statistical approaches, including machine learning methods; (iv) evaluate the capacity of climate models to simulate changes in climatic modes of variability in response to variations in external forcing; (v) strengthen links with current working groups; and (vi) expand the group to include researchers from a variety of communities (e.g. wide regional representation, experts in each mode and key period) related to the study of modes of variability. We also agreed that the edition of a special issue based on modes of climate variability would be one of the main outputs of the working group.

The workshop also included an outreach activity at CosmoCaixa (Barcelona Science Museum) to engage secondary school students in climate change (Fig. 1). A video, entitled "Climate Change: the FAQs" (youtube.com/watch?v=|-2wR9zx7Rg), was officially released during this event. The video was created by some of the co-organizers of the workshop with the aim of answering the most common questions about climate change posed by young students. A total of 180 attendees had the opportunity to watch the video and ask additional questions during a round-table discussion with a Catalonian government representative, two science communicators, a climate-change activist, and two climatologists, all of whom work on different aspects of climate change.

\section{AFFILIATIONS}

IInstitute of Earth Sciences Jaume Almera (ICTJA-CSIC), Barcelona, Spain

Institute of Environmental Assessment and Water

Research (IDAEA-CSIC), Barcelona, Spain ${ }^{3}$ School of Archaeology, Geography and Environmental Sciences, University of Reading, UK ${ }^{4}$ Department of Geography, Royal Holloway University of London, UK

${ }^{5}$ Geography Department, Durham University, UK ${ }^{6}$ Barcelona Supercomputing Center (BSC-CNS), Spain Oceanic and Continental Environments and Paleoenvironments (CNRS-EPOC), University of Bordeaux, France

\section{CONTACT}

Armand Hernández: armandhernandezh@gmail.com REFERENCES

Bastos A et al. (2016) Nat Commun 7: 10315 Jerez S et al. (2013) J Appl Meteorol Climatol 52: 2204-2224

Zubiate L et al. (2017) Q J Roy Meteorol Soc 143: 552-562 